\title{
Semi Autonomous Mine Detection System
}

\section{SPIE Defense Security and Sensing}

\author{
Doug Few \\ Roelof Versteeg \\ Herman Herman
}

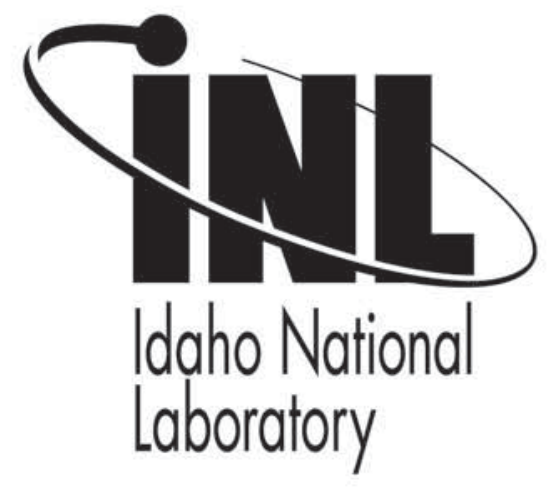

This is a preprint of a paper intended for publication in a journal or proceedings. Since changes may be made before publication, this preprint should not be cited or reproduced without permission of the author. This document was prepared as an account of work sponsored by an agency of the United States Government. Neither the United States Government nor any agency thereof, or any of their employees, makes any warranty, expressed or implied, or assumes any legal liability or responsibility for any third party's use, or the results of such use, of any information, apparatus, product or process disclosed in this report, or represents that its use by such third party would not infringe privately owned rights. The views expressed in this paper are not necessarily those of the United States Government or the sponsoring agency. 


\title{
Semi autonomous mine detection system
}

\author{
Doug Few ${ }^{\mathrm{a}}$, Roelof Versteeg ${ }^{\mathrm{a}}$ and Herman Herman ${ }^{\mathrm{b}}$ \\ ${ }^{\mathrm{a}}$ Idaho National Laboratory, 2251 N Boulevard, Idaho Falls, ID 83415; ${ }^{\mathrm{b}}$ Carnegie Mellon \\ University, National Robotics Center, Pittsburgh, PA
}

\begin{abstract}
CMMAD is a risk reduction effort for the AMDS program. As part of CMMAD, multiple instances of semi autonomous robotic mine detection systems were created. Each instance consists of a robotic vehicle equipped with sensors required for navigation and marking, countermine sensors and a number of integrated software packages which provide for real time processing of the countermine sensor data as well as integrated control of the robotic vehicle, the sensor actuator and the sensor. These systems were used to investigate critical interest functions (CIF) related to countermine robotic systems. To address the autonomy CIF, the INL developed RIK was extended to allow for interaction with a mine sensor processing code (MSPC). In limited field testing this system performed well in detecting, marking and avoiding both AT and AP mines. Based on the results of the CMMAD investigation we conclude that autonomous robotic mine detection is feasible. In addition, CMMAD contributed critical technical advances with regard to sensing, data processing and sensor manipulation, which will advance the performance of future fieldable systems. As a result, no substantial technical barriers exist which preclude - from an autonomous robotic perspective - the rapid development and deployment of fieldable systems.
\end{abstract}

Keywords: Countermine, robotics, real-time, data processing

\section{INTRODUCTION}

The CMMAD (Countermine, Mobility, Marking, Autonomy and Detection) effort is an INL led effort which is a part of the Project Manager for Countermine and Explosive Ordinance Disposal (PM-CM \& EOD) Autonomous Mine Detection System (AMDS) Risk Reduction Program. The AMDS program seeks to produce an autonomous mine detection vehicle that achieves the same performance of a handheld mine detector. The primary focus of the AMDS program is on the development of novel sensors and data processing algorithms capable of detecting mines which in future phases of this program can be integrated on existing and future robotic platforms. A secondary focus is the reduction of risk associated with the integration of such sensors on robotic vehicles. The latter was addressed through the CMMAD effort, which had the objective to reduce risk for future AMDS phases by assessing the feasibility of autonomous countermine systems through the evaluation of five Critical Interest Functions (CIF). These Critical Interest Functions, which were identified by the government as being essential for robotic autonomous mine detection, are:

1. Plug-n-Play: to what extent can control systems, sensors and manipulators easily be swapped between different robotic platforms?

2. Autonomy: to what extent can autonomous countermine systems successfully locate and avoid mines?

3. Situational Awareness: to what extent can operators of autonomous countermine systems successfully deploy and interact with such systems?

4. Platform Mobility: to what extent can robotic countermine systems successfully traverse a range of terrains?

5. Marking: to what extent can robotic countermine systems accurately and reliably mark mines and safe paths?

A challenge faced by the INL led team was that investigation of these critical interest functions requires several physical instantiations of an AMDS system. As no such AMDS systems are currently available, several system instances were

Proceedings of 2010 SPIE Defense, Security and Sensing Meeting. Conference 7664: Detection and Sensing of Mines, Explosive Objects and Obscured Targets XV. Paper 7664-44. Cite as: Few, D, Versteeg, R. and Herman, H. (2010) Semi autonomous mine detection system, Proc. SPIE 7664, 7664-44 
designed and constructed for the CMMAD effort. While these systems (hereafter referred to as a CMMAD systems) do not have the advanced sensors which are likely to be used in fielded AMDS systems, they have the essential components which allow for the investigation of the CIFs. In the remainder of this paper we discuss the general structure of AMDS systems and the architecture and performance of our CMMAD systems.

\section{SYSTEM ARCHITECTURE}

\subsection{AMDS and CMMAD system architecture}

As mentioned above, several CMMAD systems were designed and built during the CMMAD effort. The architecture of these systems is based on a consideration of the likely configuration of the future AMDS systems. Specifically, such systems (figure 1) will likely consist of a robotic vehicle equipped with sensors required for navigation and marking, one or more countermine sensors (likely having both GPR and EMI sensing modalities), a countermine sensor manipulator, and a number of integrated software packages which would provide for real time processing of the countermine sensor data as well as control of the robotic vehicle. Such a system would likely also comprise an Operator Control Unit (OCU) which will allow users to interface to the robot.

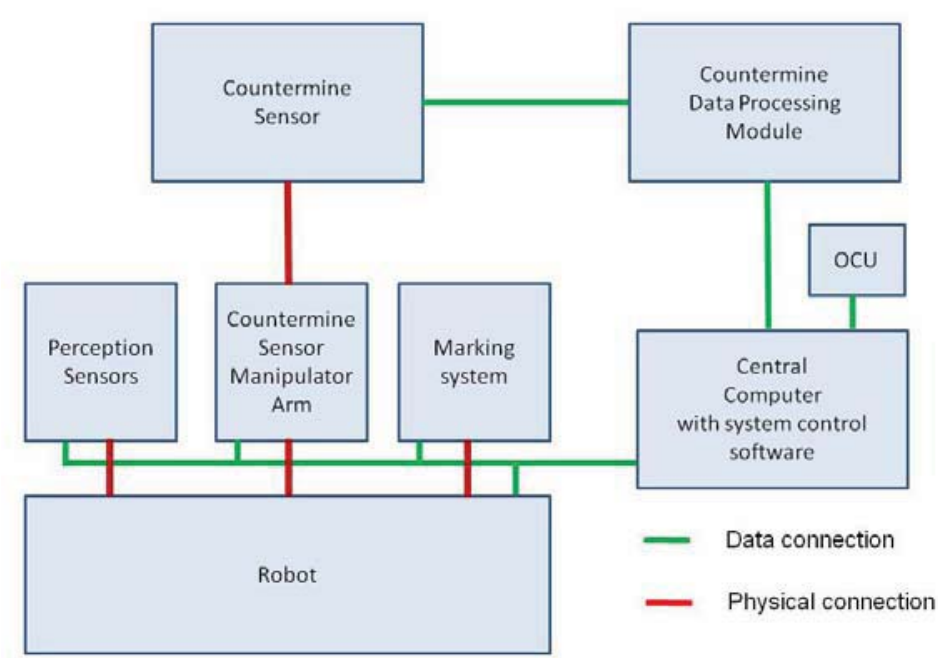

Figure 1. Block Diagram of likely AMDS System architecture. Red lines show physical connections. Green lines indicate data connections.

The CMMAD systems which were constructed as part of the risk reduction effort are both similar to and different from the future fielded AMDS systems. Future fielded AMDS systems will most likely use significantly more advanced countermine sensors, actuators with more degrees of freedom (DOF), novel generation robots, and different perception sensors. In addition, such systems will likely have very different user interfaces than the current CMMAD systems.

However, future AMDS systems will be similar in terms of the requirements, the type of components and the architecture. In terms of requirements, both the CMMAD and AMDS systems need to be able to navigate autonomously, detect and mark mines in real time, and avoid mines and above ground obstacles. In terms of types of components, both the AMDS and CMMAD systems will likely consist of a robotic vehicle equipped with sensors required for navigation and marking, an OCU for tasking and visualization, one or more countermine sensors (likely having both GPR and EMI sensing modalities), a countermine sensor manipulator, and a number of integrated software packages which provide for real time processing of the countermine sensor data as well as control of the robotic vehicle. This leaves the architecture, with which we here connotate the high level functionality and interplay of the components of the hardware and software. We designed the architecture of CMMAD system so it can be applied to the AMDS systems as well. Again - while the underlying hardware, software and implementation of the functionality may be radically different between the AMDS system and the CMMAD system, the architecture that we developed for CMMAD should be applicable to AMDS. 


\section{SYSTEM SOFTWARE}

\subsection{CMMAD Software components overview}

The CMMAD systems (as well as future AMDS systems) require several distinct software components which operate in an integrated manner through well defined interfaces. These components are (1) Software which orchestrates and controls the system and orchestrates the interaction between the components of the system (2) OCU software (i.e. the software which runs on the Operator Control Unit hardware (typically a computer with Windows software)) and (3) Countermine data processing software.

For the CMMAD system implemented by INL, the first component is the RIK. The second component is the INL developed OCU package, and the third component is the MSPC (Mine Sensor Processing Code).

The last two components (the OCU and the MSPC) have clearly defined roles. However, the first component (the RIK) has to perform and coordinate a multitude of roles. These include (1) Hardware control role: Control the behavior of the physical hardware (robot, marking system, sensor manipulator arm), (2) Mapping role: Receive and process the data from the perception sensors to build up a representation of the environment using SRI International's KARTO laser based Simultaneous Localization and Mapping (SLAM) software package [1, 2], (3) Coordinator role: Data fusion, management and abstraction as well as orchestration of communication between all the components and (4) Autonomy role: path planning and execution, obstacle avoidance and mine detection specific behaviors (second look, marking and avoidance).

\subsection{RIK overview}

Starting in 2001 INL researchers have been developing a robotic control framework. One of the design premises used during the development of the robot control framework was that a set of robot capabilities is generic to all unmanned ground vehicle tasks. Regardless of use domain, an unmanned vehicle must be able to detect and avoid obstacles, build data representations, plan paths, and accept a variety of inputs consistent with task demands. The framework has been going through an iterative development cycle where performance and behaviors have been iteratively developed in the laboratory in response to user needs and field evaluated and tested by INL and external users[3, 5]. The framework (Figure 2) consists of: (1) a robot-sensor architecture for interfacing a variety of robot platforms, perceptual sensors, and algorithmic capabilities, (2) a communications server for sending and receiving messages to trigger capabilities via the operator control unit and external processes, and (3) an application layer that consists of task level and interaction behaviors for intelligent unmanned ground vehicle navigation.

This software framework, called the Robot Intelligence Kernel (RIK), is being used throughout the robotics community to bootstrap intelligent ground robotics efforts and was used as the starting point for the CMMAD system control software.

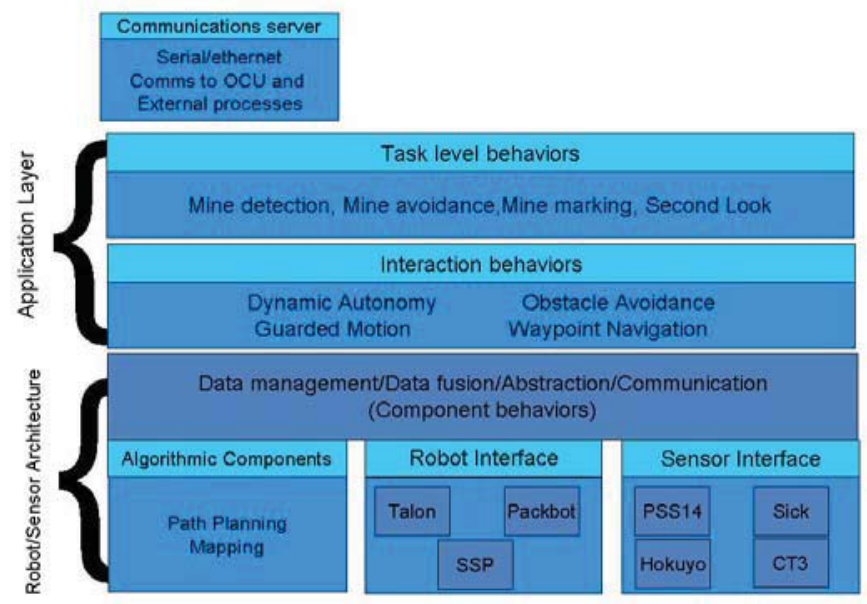

Figure 2. Schematic of the RIK showing robot sensor architecture, application layer and communications server and their components. 
At the beginning of the CMMAD effort the RIK provided a well tested and extensive suite of generic capabilities which had been developed for and applied to a number of intelligent ground robot missions. However, in order to create an operational instance of an autonomous countermine system which used realistic components to investigate and answer the CMMAD critical interest functions, modifications, improvements and enhancements were made to each of the three RIK subcomponents. Specifically in order to investigate the CMMAD Critical Interest Functions, we need to develop new capabilities within the RIK. The foundations of these capabilities are new sensors, actuators, behaviors and underlying software components. We discuss here the novel behaviors required to perform the autonomous countermine mission which were added to the RIK.

\subsection{RIK Behaviors}

Within the RIK, three different types of behaviors are recognized. These are task level behaviors, tactical behaviors and component level behaviors. Task level behaviors are a category of coordinated robot-sensor-actuator behaviors that are the product of a task analysis breakdown of the application domain or mission space the robot is being deployed in. The task level behaviors tie together both the tactical and component behaviors defined below into an intelligent series of high and low level events to accomplish a phenomenon. New task level behaviors required for the CMMAD effort included, for instance "detect, mark and avoid mines" and "detect and mark mines in overpass mode".

Tactical behaviors are the class of behaviors that are associated with the different interaction modes for robotic system navigations. Four robot modes of tactical control are available for the RIK. These modes correspond to increasingly higher levels of robot autonomy. They are (Figure 3)

- Tele Mode: a fully manual mode of operation, in which the operator must manually control robot movement.

- Safe Mode: this is similar to Tele Mode, in that robot movement is dependent on manual control. However, in Safe Mode, the robot is equipped with a level of initiative that prevents the operator from colliding with obstacles by utilizing range sensors to detect the systems proximity to obstacles.

- Shared Mode: in this mode the robot can relieve the operator from the burden of direct control, using reactive navigation to find a path based on perception of the environment. Shared Mode provides for a dynamic allocation of roles and responsibilities. The robot accepts varying levels of operator intervention and supports dialogue through the use of a finite number of scripted suggestions (e.g.,"Path blocked! Continue left or right?") and other text messages that appear in a text box within the graphical interface.

- Collaborative Tasking Mode: In this mode the robot autonomously performs all decision making and navigation. The only effort that the user provides is at the tasking level.

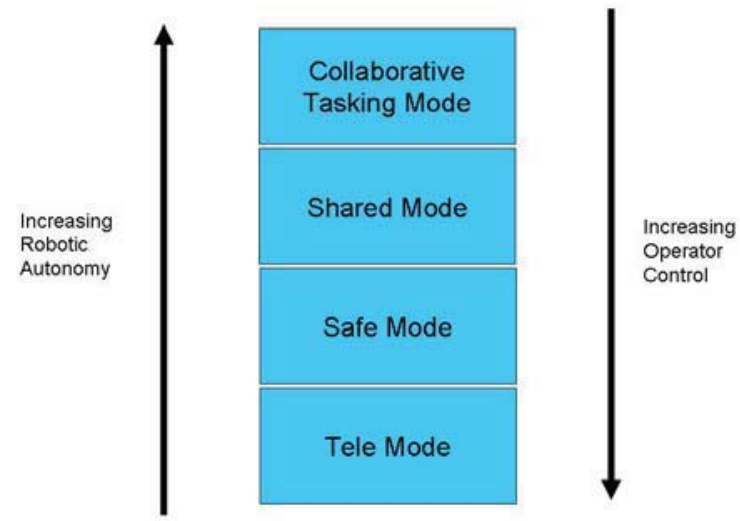

Figure 3 Different modes of robotic behavior. Note that the robot is always in one of these modes, and that these modes are mutually exclusive. Also note that both the robot and the operator can change modes. However, the robot can only lower the autonomy (for instance, when the robot is not able to execute a mission, it will pass control to the operator by going for example from shared mode to safe mode)

These RIK interaction modes (and the underlying and associated behaviors) have empirically shown the ability to reduce remote operator workload and improve system user performance when used for search and detection or reconnaissance 
missions[3,4,6]. While the different interaction modes remained the same, the underlying implementation of the associated tactical behaviors was expanded significantly to accommodate the countermine mission. One example of this was the inclusion of mines as obstacles in both the obstacle avoidance and path planning behavior. This is necessary since the robots used in CMMAD do not have overpass capability, so they have to avoid going over the detected mines to prevent triggering them.

Component level behaviors are behaviors which are used by the task level and the tactical behaviors. They are typically behaviors which can be abstracted (such as calibrate sensor, detect mines, mark a mine). These behaviors are all required to fulfill the task level and tactical behaviors. While they require a hardware specific implementation, as these tasks are abstracted it is relatively simple to enhance the underlying implementation while leaving the task level behaviors the same.

A typical component behavior is an asynchronous process that is the result of a series of coordinated events between the robotic platform and a sensor or actuator defined within the robot-sensor architecture. An example of a component behavior which was implemented under CMMAD is the mine sensor calibration. This requires a series of sensor switch manipulations, SSP (Sensor Scanner Payload) actuations, and robot movements into a coordinated series of events that mimic human actions used to perform the calibration routine. Other component behaviors which were implemented include for example the marking behavior (which require a coordination between the robot, the SSP and the marking system), the detection and the terrain following behavior (which requires an interplay between the scrolling height map and the SSP).

\subsection{Operator Control Unit (OCU)}

The OCU (Operator Control Unit) is a standalone component and provide the membrane for human interaction with an RIK enabled robotic system. With RIK enabled robotic systems, the intelligent control capabilities reside on the robotic platform itself. The OCU communicate with a RIK enabled platform via the RIK Communications Server to offer the ability for users to command and query the robotic platform. In the case of the CMMAD system, the OCU consists of a 3D graphical user interface [7] which runs on a standard PC with Windows operating systems. The 3D graphical use interface provides methods for commanding the robot platform utilizing any of the levels of autonomy described above. Screenshots of the OCU from field testing effort of the CMMAD systems are shown in figure 4.
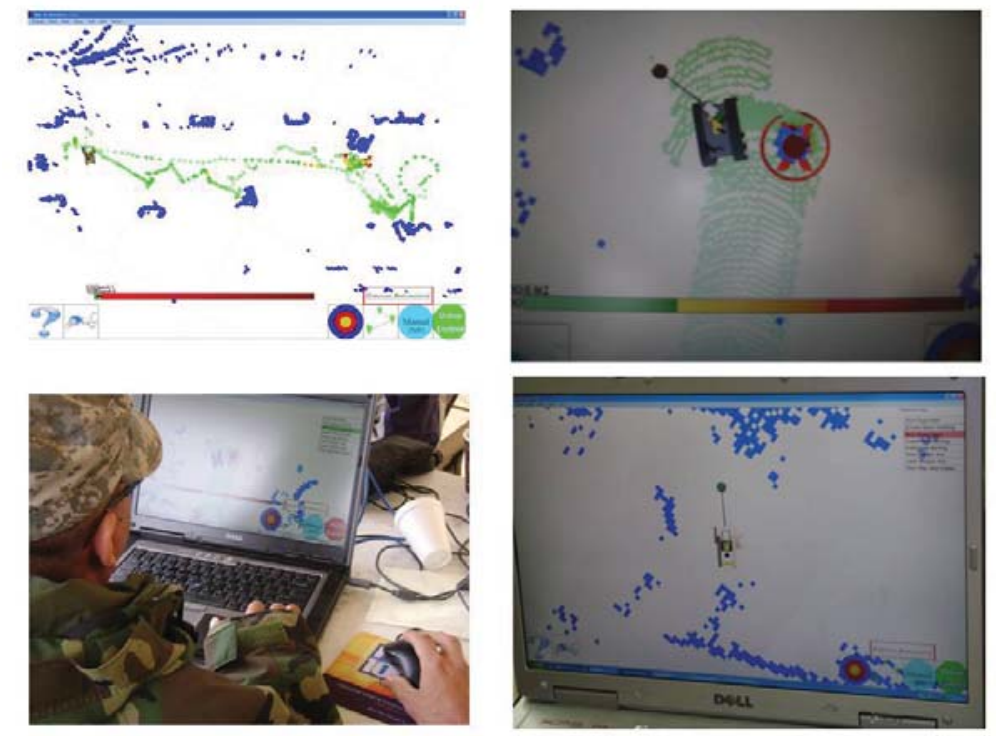

Figure 4 Screen shot of the OCU during different missions, showing control of the robot using a tasking target with waypoints, hazard map, and sensor readings. (SHOULD BE ENLARGED A LITTLE BIT AND ANNOTATED)

\subsection{Mine Sensor Processing Code}

Mine detection and characterization was not the focus of CMMAD. However, in order to be able to address the CIF the CMMAD system needs to be able to do real time data processing of threat sensor data. This required the INL team to 
design and implement an approach to realtime autonomous robotic mine detection. This implementation is called the MSPC (Mine Sensor Processing Code)

Development of the MSPC can roughly be divided in two elements. First, we designed and implemented a generic, algorithm (and to a large extent sensor agnostic) software framework (Figure 5) which allows for communication with the RIK using a content rich, well defined protocol, and which allows for data ingestion, data management (ie maintaining relevant data in memory in a rapidly accessible map, and discarding the data when it is no longer needed) and spatial map generation (for display of "raw" sensor readings in the OCU interface as the robotic system progresses).

Second, within this framework we implemented a simple prescreener, classifier and centroid calculator were implemented. The prescreener uses the real time calculated statistics of datastream to decide whether a prescreener hit is warranted. Once a prescreener hit has been declared, the characterization module is engaged. This performs pre processing of the datastream (in a manner similar to that discussed in [8], [9] and [10] and subsequent to this uses a standard neural network (trained on a dataset with known threat locations) to determine the confidence of a signal being associated with a threat). The results of this classification are used by a NN based centroid calculator to determine if a centroid exist, and if so, if this centroid is stable. Once a stable centroid has been found it is communicated to the RIK, who then takes appropriate action. It should be noted that because of the modular software structure of the MSPC it is trivial to either accommodate other sensor data than the one used in the CMMAD effort, or to accommodate different prescreeners, threat classifiers or centroid calculators.

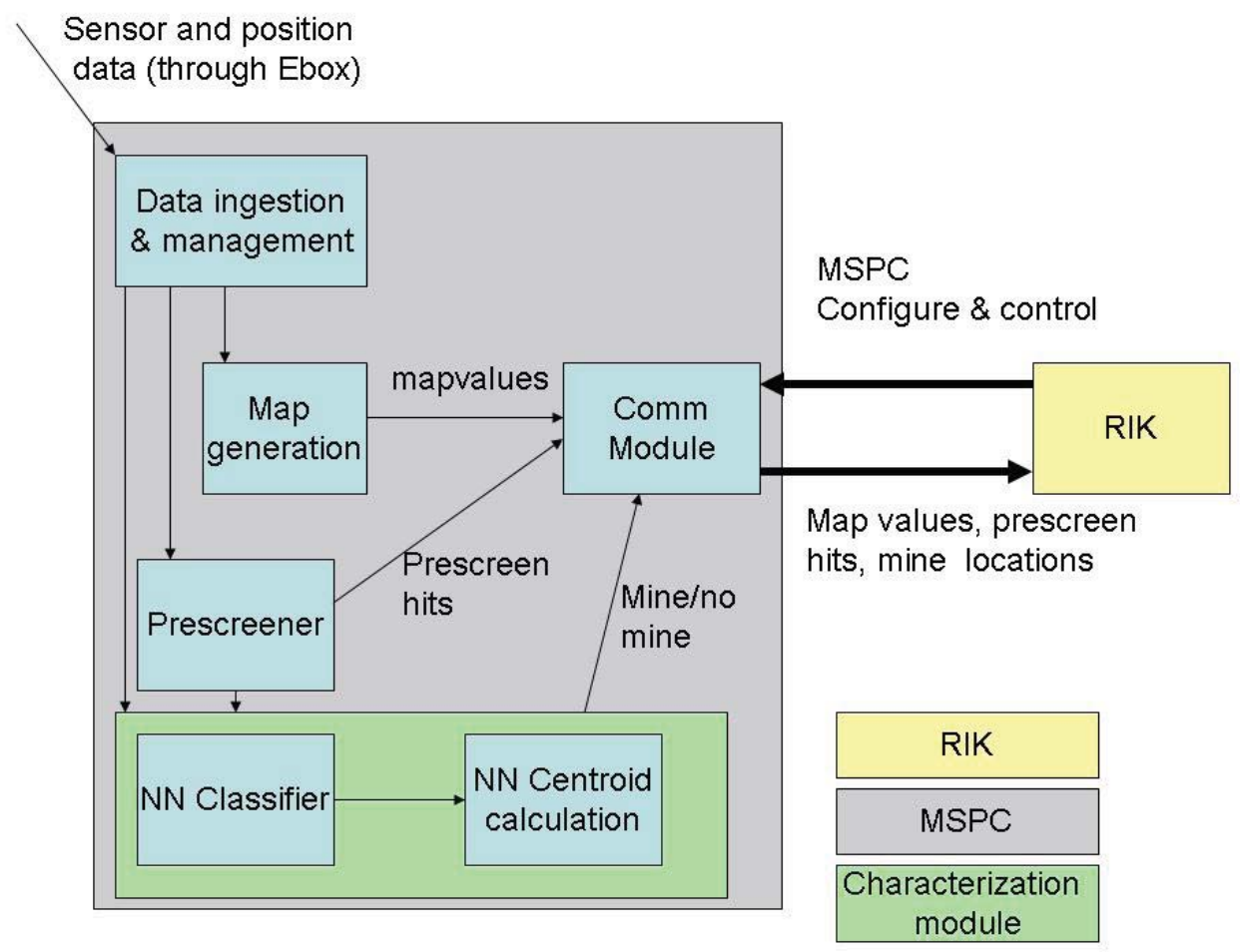

Figure 5 Flowdiagram of MSPC and its interaction with the RIK. Within the RIK the characterization module performs the actual characterization

\section{CMMAD SYSTEMS}

Six different physical systems were used during the CMMAD effort (table 1).These systems differ in robotic platform, countermine sensor and position sensors and were used to investigate different aspects of each of the critical interest functions 
Table 1: Overview of the Different CMMAD System Configurations

\begin{tabular}{|c|c|c|c|c|c|c|c|}
\hline System name & Robot & $\begin{array}{l}\text { RIK on } \\
\text { board }\end{array}$ & $\begin{array}{l}\text { Threat } \\
\text { Sensor }\end{array}$ & $\begin{array}{l}\text { SSP (Sensor Scanner } \\
\text { Package) }\end{array}$ & $\begin{array}{l}\text { E-Box (Interface } \\
\text { and Embedded } \\
\text { Computer) }\end{array}$ & $\begin{array}{l}\text { Perception } \\
\text { sensors }\end{array}$ & $\begin{array}{l}\text { Position } \\
\text { sensors }\end{array}$ \\
\hline NIITEK system & $\begin{array}{l}\text { Foster } \\
\text { Miller } \\
\text { Talon }\end{array}$ & Yes & $\begin{array}{l}\text { NIITEK } \\
\text { CT3 }\end{array}$ & No & No & No & IMU GPS \\
\hline $\begin{array}{l}\text { Spawar } \\
\text { Surrogate Talon }\end{array}$ & $\begin{array}{l}\text { Foster } \\
\text { Miller } \\
\text { Talon }\end{array}$ & No & None & $\begin{array}{l}\text { Simulated SSP (same } \\
\text { formfactor/weight) }\end{array}$ & No & No & IMU GPS \\
\hline $\begin{array}{l}\text { Spawar } \\
\text { Surrogate } \\
\text { Packbot }\end{array}$ & $\begin{array}{l}\text { Irobot } \\
\text { Packbot }\end{array}$ & No & None & $\begin{array}{l}\text { Simulated SSP and } \\
\text { simulated FIDO (same } \\
\text { formfactor/weight) }\end{array}$ & No & No & IMU GPS \\
\hline $\begin{array}{l}\text { Countermine } \\
\text { Talon }\end{array}$ & $\begin{array}{l}\text { Foster } \\
\text { Miller } \\
\text { Talon }\end{array}$ & Yes & $\begin{array}{l}\text { Cyterra } \\
\text { ANPSS14 }\end{array}$ & Yes & Yes & $\begin{array}{l}\text { Hokuyo }+ \text { Sick } \\
\text { Rangefinders }\end{array}$ & $\begin{array}{l}\text { Encoders } \\
+ \text { IMU }\end{array}$ \\
\hline $\begin{array}{l}\text { Explosive } \\
\text { detection Talon }\end{array}$ & $\begin{array}{l}\text { Foster- } \\
\text { Miller } \\
\text { Talon }\end{array}$ & Yes & $\begin{array}{l}\text { ICX } \\
\text { Nomadics } \\
\text { FIDO }\end{array}$ & Yes & Yes & $\begin{array}{l}\text { Hokuyo }+ \text { Sick } \\
\text { Rangefinders }\end{array}$ & $\begin{array}{l}\text { Encoders } \\
+ \text { IMU }\end{array}$ \\
\hline $\begin{array}{l}\text { Countermine } \\
\text { Packbot }\end{array}$ & $\begin{array}{l}\text { Irobot } \\
\text { Packbot }\end{array}$ & Yes & $\begin{array}{l}\text { Cyterra } \\
\text { ANPSS14 }\end{array}$ & Yes & Yes & $\begin{array}{l}\text { Hokuyo }+ \text { Sick } \\
\text { Rangefinders }\end{array}$ & $\begin{array}{l}\text { Encoders } \\
+ \text { IMU }\end{array}$ \\
\hline $\begin{array}{l}\text { Explosive } \\
\text { detection } \\
\text { Packbot }\end{array}$ & $\begin{array}{l}\text { Irobot } \\
\text { Packbot }\end{array}$ & Yes & $\begin{array}{l}\text { ICX } \\
\text { Nomadics } \\
\text { FIDO }\end{array}$ & Yes & Yes & Hokuyo + Sick & $\begin{array}{l}\text { Encoders } \\
+ \text { IMU }\end{array}$ \\
\hline
\end{tabular}

In the remainder of this paper we focus on the CMMAD systems which have both the RIK and SSP subsystem on board to allow for obstacle avoidance. The SSP subsystem includes threat sensor, embedded interface and computational electronic, perception sensors and position sensors. This includes the countermine Talon and countermine Packbot.

\subsection{Countermine systems}

Two "complete" CMMAD systems were constructed. These systems are complete in the sense that they have both the RIK on board as well as countermine and perception sensors on board. These include the countermine Talon and the countermine Packbot. The countermine Talon (Figure 6a) is a stock Talon equipped with the CMU SSP subsystem, which contains the interface electronic, embedded computer, SICK and Hokuyo lasers, the SPAWAR marking system and the Cyterra AN/PSS14 sensor. The countermine Packbot (Figure 6b) is a stock Packbot equipped with the CMU SSP subsystem, which includes SICK and Hokuyo lasers, a camera and the Cyterra AN/PSS14 sensor. The countermine Packbot and the countermine Talon were used for the investigation of the autonomy, situational awareness, plug and play and marking CIF. As the CMU SSP are discussed in a companion paper [11] we do not discuss these components here.

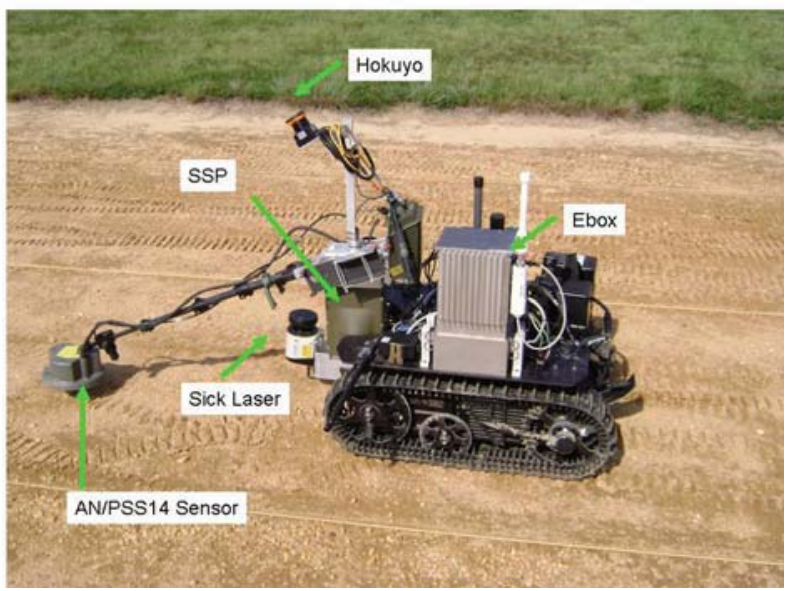

(a)

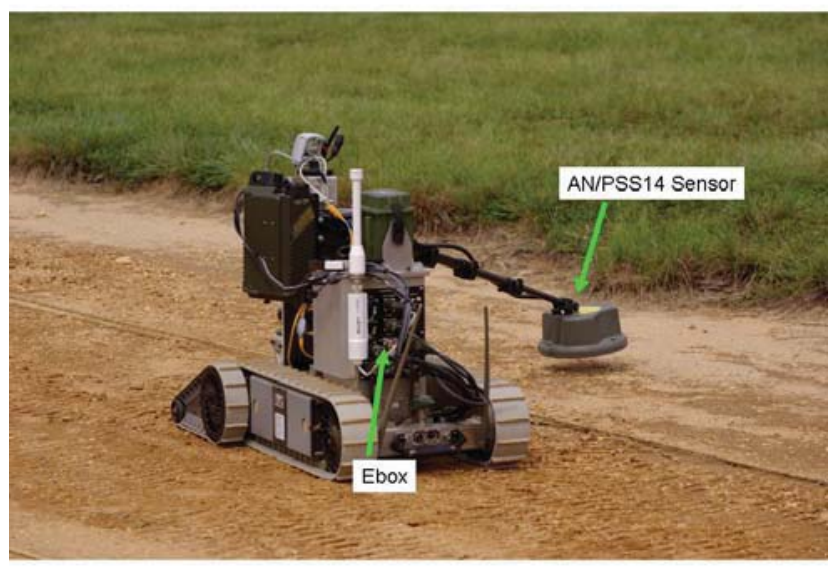

(b) 
Figure 6 (left) Countermine Talon. (b) Countermine packbot

\section{SYSTEM TESTING RESULTS}

The CMMAD systems were used to investigate the critical interest functions. This was done both in a software testing environment, at an INL mine field testing area which contained high metal AT mine and at a government test site. Testing for the mobility CIF was done at another government test site using the SPAWAR Surrogate Talon and Packbot (Table 1). The software testing is done by hardware in the loop approach in which we used both real and simulated hardware components (table 2). This hardware in the loop approach was essential in system development. Note that the software package used (ie the RIK, OCU and MSPC software) is the same for all configurations.

\begin{tabular}{|c|c|c|}
\hline Robot Type & Sensor Scanner Payload Type & Sensor Data Source \\
\hline Simulated & Simulated & Simulated \\
\hline Simulated & CMMAD SSP & Simulated \\
\hline Talon/Packbot & Simulated & Simulated \\
\hline Talon/Packbot & CMMAD SSP & PSS 14 \\
\hline Talon/Packbot & CMMAD SSP & Simuled \\
\hline
\end{tabular}

Table 2. Matrix of configurations which are supported by the RIK architecture. These configurations were used both in development and CIF investigation

The testing results discussed here focus on the tests for the autonomy CIF, which is the primary CIF. Results of the other CIF assessments were successful, but will be discussed in future publications and reports.

The autonomy CIF asks whether robotic systems can successfully autonomously navigate terrains and locate and avoid mines. Associated with and underlying this question is an implicit mental model on the operation and deployment of such a system. This mental model is that an operator would task a robotic system to execute a specific mission, and that the operator would have real time feedback (through a visual display and/or line of sight to the system) as to the mission progress and status and would have the ability to modify the mission based on this feedback. Modifications could include overriding the robots classification, retasking the robot, or changing the planned path based on observations beyond the robots perception range. From an end user perspective, the answers to the autonomy CIF will result from a mission level assessment (i.e. can the system find mines, path plan, navigate the terrain and avoid obstacles). However, from a risk reduction perspective it is important - in addition to this mission level assessment - to have an understanding of the components and interplay which enables this autonomy as well as the interplay between the robot and the operator. To investigate the CMMAD autonomy CIF, we implemented new task level and component behaviors, and implemented interaction behaviors between the MSPC and the RIK. By doing this within the RIK architecture, we assured that the efforts done under CMMAD can be extended easily to include new robots, actuators, mine detection sensors or classification algorithms.

Below we show here results from our mixed real and simulated environment which demonstrates the overall performance. A similar performance was obtained in the field but is not shown here. These results (shown here through a series of screenshots from the OCU) show results from a mine detection mission utilizing an iRobot Packbot, the CMMAD SSP and simulated mine data. Note that from a result perspective the simulated sensor data mirrors what was observed at the government test site using the Countermine Talon. 


\section{Autonomous Countermine Mission Sequence}

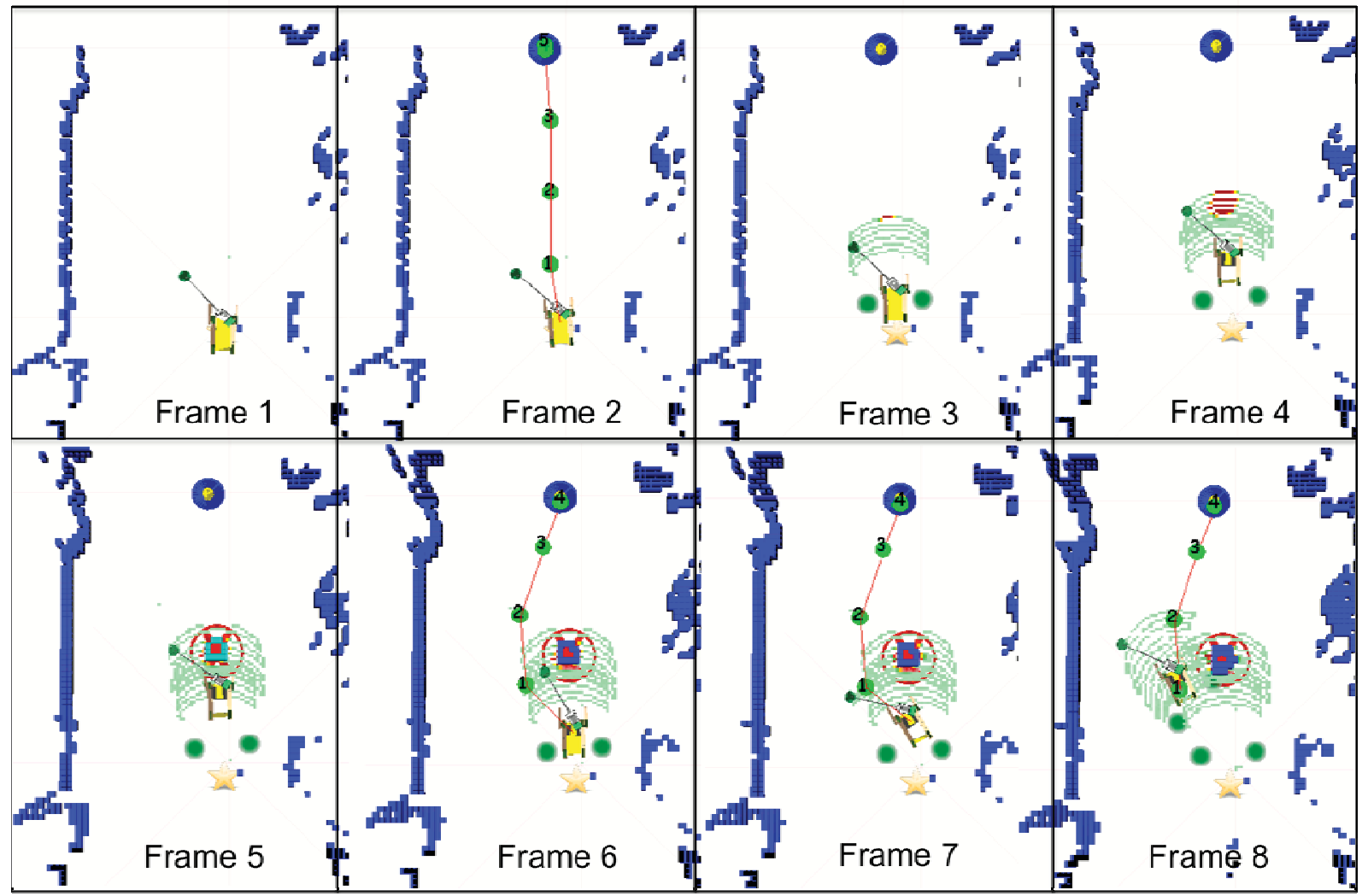

Figure 7 Series of screenshots showing progress of autonomous countermine mission

The system operates as follows:

Frame 1: At the start of the countermine mission, the sensor has been calibrated by the system operator and is waiting tasking by the operator.

Frame 2: the system operator has given the robot a goal, the robot's path planning algorithm analyzed the environment and calculated the interstitial waypoints for achieving the operators goal.

Frame 3: The MSPC prescreener has triggered (in this case on an EMI signal above the threshold (calculated based on calibration data) and communicates a prescreener message to the robot. Upon receiving the prescreen hit, the robot retracts the goal point and launches a second-look behavior.

Frame 4: the robot is characterizing the subsurface during the second look behavior. Note that the sensor scan width has widened and the EMI data resolution has become denser.

Frame 5: The MSPC classified a mine location and articulated the mine location to the robot. The robot exits the second look behavior, communicates the mine location to the OCU and adds the mine as a virtual obstacle. The robot will mark the mine location at this point.

Frame 6: With the mine located and marked the operator's original goal point is re-established. The path planning algorithm identified the added mine location and calculated a series of waypoints around the detected mine location.

Frames $7 \&$ 8: The robot is continuing with the countermine mission following the mine detection event.

These results show an example of a successful autonomous performance of the robotic countermine system. In addition to the described capabilities, there are obviously numerous other aspects (such as sensor terrain following, environmental sensing and positioning) which are required. Most of these have been addressed successfully in other efforts (both by the INL group and others) and will not be discussed here. 


\section{DISCUSSION AND CONCLUSION}

The CMMAD effort was a risk reduction effort for the AMDS program. To this end, CMMAD addressed five critical interest functions related to robotic mine detection efforts: (1) Plug-n-Play, (2) Autonomy, (3) Situational Awareness (4) Platform Mobility and (5) Marking. The main conclusion of the CMMAD investigation into the critical interest functions is that autonomous robotic mine detection is feasible. Technical advances with regard to sensing, data processing and sensor manipulation will advance the performance of fieldable systems. We have also found no substantial technical barriers that will preclude - from an autonomous robotic perspective - the development of fieldable systems. However, this conclusion is predicated on several assumptions about the design and implementation of such systems. To some extent these assumptions are implicit in the systems presented here. We briefly discuss some of these assumptions here.

\subsection{Plug and play}

Component plug and play will only be possible if components meet very explicitly developed specifications for electrical, mechanical and software interfaces. This raises the questions as to whom is responsible for developing and maintaining these interface specifications. Given the current plethora of manufacturer specific (and often semi proprietary and minimally documented interfaces) it seems unlikely that such a standard will develop organically without government guidance. Additionally, it is important to note that the current standards efforts like JAUS and STANAG 4586 currently lack the level of detail in their respective specifications to implement the plug and play capability as demonstrated as part of this evaluation. Thus, any future effort which seeks to develop modular autonomous countermine systems should consider the need for coordinated electro-mechanical interfaces on disparate robotic platforms as well as influencing the existing robotic interoperability standards to support run-time system configuration specification.

\subsection{Autonomy and performance specifications.}

Autonomy was a central theme of the CMMAD effort, and the CMMAD team feels that the conclusions obtained in the autonomy chapter stand on their own. However, one of the observations made by the team during the execution of the CMMAD project was that the interpretation of what autonomy actually means in the framework of autonomous mine detection systems is subject to a significant amount of interpretation. Or, it seems that a clear agreement on what is meant by an autonomous system (both from a user and developer perspective) is necessary in any future AMDS effort. Or, a necessary step in a further effort is a clear definition of the performance specifications for an AMDS system. While the current risk reduction effort has shown that all the components exist to make a fieldable system, one obvious observation is that any specific instance of a system will have a specific performance envelope that is optimized for a set of applications. In another word, it is unlikely to develop a system which does well in all possible circumstances. For instance, systems can be made which perform really well in investigating a wide open area relatively fast, or systems which are really nimble, or systems which make an effective use of GPS, or work well in an indoor environment or in a low contrast outdoor environment. However, it will be hard to develop a system which functions equally well in all environments. The solution to this could be to have components which are tailored to perform well in specific environments, so that users could "assemble" different systems dependent on their needs, but even so, clear agreement on expectations is needed to proceed.

\section{ACKNOWLEDGEMENTS}

Funding for the CMMAD effort was provided through the AMDS program which was administered through US Army RDECOM CERDEC Night Vision and Electronic Sensors Directorate). We acknowledge the support and guidance of the program manager (Ed McAuley) as well as that of the NightVision team (Mark Locke, Eloisa Lara and Matt Aiello) and the IDA team. The support of multiple staff members at INL and in the different institutions and organizations participating in the CMMAD effort is gratefully acknowledged. 


\section{REFERENCES}

[1] K. Konolige. "Large-scale map-making," In Proc. of the AAAI, San Jose, CA, 2004, pp. 457-463.

[2] J.S Gutman and K. Konolige. "Incremental Mapping of Large Cyclic Environments," In proceedings of the CIRCA 2000, 1999.

[3] H. A. Yanco, B. Keyes, J. L. Drury, C. W. Nielsen, D. A. Few, D. J. Bruemmer. "Evolving Interface Design for Robot Search Tasks" In the Journal of Field Robotics. Volume 24 (8/9) pp 779-799, 2007

[4] D.J. Bruemmer, D.A. Few, R.L. Boring, J.L. Marble, M.C. Walton, and C. W. Nielsen. "Shared Understanding for Collaborative Control" In IEEE Transaction on Systems, Man, and Cybernetics, Part A: Systems and Humans. Volume 35 Number 4, pp 494-504 July 2005

[5] D. A. Few, D.J. Bruemmer, M.C. Walton. "Improved Human-Robot Teaming through Facilitated Initiative," In Proc. of the IEEE International Symposium on Robot and Human Interactive Communication, Hatfield, United Kingdom 6-8 Sep 2006

[6] D. J. Bruemmer, D. A. Few, C. W. Nielsen, M. C. Walton. "Intelligent Behaviors for Landmine Detection and Marking. In the Journal of Mine Action, Issue 11.2 pp 97-102 Spring 2008

[7] D. J. Bruemmer, D.A. Few, M. C. Walton, R. L. Boring, J. L. Marble, C. Nielsen, and J. Garner. "Turn off the television!": Real-world robotic exploration experiments with a virtual 3-D display. To appear in Proceedings of the Hawaii International Conference on System Sciences (HICSS) 2005

[8] J.-X. Liu, B. Zhang, R.-B. Wu, "GPR Ground Bounce Removal Methods Based on Blind Source Separation," Progress in Electromagnetics Research Symposium, pp. 26-29, Cambridge, USA, March 2006.

[9] P. D. Gader, W.-H. Lee, J. N. Wilson, "Detecting Landmines With Ground-Penetrating Radar Using Feature-Based November

Rules, Order Statistics, and Adaptive Whitening," IEEE Trans. Geosci. Remoter Sens., vol. 42. no. 11,

[10] J. N. Wilson, P. D. Gader, K. C. Ho, W.-H.Lee, R. J. Stanley, T. C. Glenn, "Region Processing of Ground Penetrating Radar and Electromagnetic Induction for Hanheld Landmine Detection," Proceedings of SPIE, Vol. 5415, pp. 933- 944, Apr. 2004.

[11]H.Herman, D.Few, R.Versteeg,. J.Valois,. J.McMahill, M. Licitra, E. Henciak "Modular Countermine package for small robots". in 2010 SPIE Defense Security and Sensing Meeting. 2010. Orlando, Florida. 